\title{
On the Construction of Analytic-Numerical Approximations for a Class of Coupled Differential Models in Engineering
}

\author{
Emilio Defez ${ }^{1}$, Vicente Soler ${ }^{2}$, Roberto Capilla ${ }^{3}$ \\ ${ }^{1}$ Instituto de Matemática Multidisciplinar, Universitat Politècnica de València, Valencia, Spain \\ ${ }^{2}$ Departamento de Matemática Aplicada, Universitat Politècnica de València, Valencia, Spain \\ ${ }^{3}$ Departamento de Ingeniera Electrónica, Universitat Politècnica de València, Valencia, Spain \\ Email: edefez@imm.upv.es, vsoler@mat.upv.es, rcapilla@eln.upv.es
}

Received 7 October 2014; revised 1 November 2014; accepted 3 December 2014

Academic Editor: Antonio Hervás Jorge, Department of Applied Mathematics, Universidad Politécnica de Valencia, Spain

Copyright (C) 2015 by authors and Scientific Research Publishing Inc.

This work is licensed under the Creative Commons Attribution International License (CC BY).

http://creativecommons.org/licenses/by/4.0/

(c) (i) Open Access

\section{Abstract}

In this paper, a method to construct an analytic-numerical solution for homogeneous parabolic coupled systems with homogeneous boundary conditions of the type $u_{t}=A u_{x x}$, $A_{1} u(0, t)+B_{1} u_{x}(0, t)=0, A_{2} u(1, t)+B_{2} u_{x}(1, t)=0,0<x<1, t>0, u(x, 0)=f(x)$, where $A$ is a positive stable matrix and $A_{1}, A_{2}, B_{1}, B_{2}$ are arbitrary matrices for which the block matrix $\left(\begin{array}{ll}A_{1} & B_{1} \\ A_{2} & B_{2}\end{array}\right)$ is non-singular, is proposed.

\section{Keywords}

Coupled Diffusion Problems, Coupled Boundary Conditions, Vector Boundary-Value Differential Systems, Sturm-Liouville Vector Problems, Analytic-Numerical Solution

\section{Introduction}

Coupled partial differential systems with coupled boundary-value conditions are frequent in different areas of science and technology, as in scattering problems in Quantum Mechanics [1]-[3], in Chemical Physics [4]-[6], coupled diffusion problems [7]-[9], modelling of coupled thermoelastoplastic response of clays subjected to 
nuclear waste heat [10], etc. The solution of these problems has motivated the study of vector and matrix SturmLiouville problems, see [11]-[14] for example.

Recently [15] [16], an exact series solution for the homogeneous initial-value problem

$$
\begin{gathered}
u_{t}(x, t)-A u_{x x}(x, t)=0, \quad 0<x<1, \quad t>0 \\
A_{1} u(0, t)+B_{1} u_{x}(0, t)=0, \quad t>0 \\
A_{2} u(1, t)+B_{2} u_{x}(1, t)=0, \quad t>0 \\
u(x, 0)=f(x), \quad 0 \leq x \leq 1
\end{gathered}
$$

where $u=\left(u_{1}, u_{2}, \cdots, u_{m}\right)^{T}$ and $f(x)=\left(f_{1}(x), f_{2}(x), \cdots, f_{m}(x)\right)^{T}$ are a $m$-dimensional vectors, was constructed under the following hypotheses and notation:

1. The matrix coefficient $A$ is a matrix which satisfies the following condition

$$
\operatorname{Re}(z)>0, \quad \forall z \in \sigma(A)
$$

where $\sigma(C)$ denotes the set of all the eigenvalues of a matrix $C$ in $\mathbb{C}^{m \times m}$. Thus, $A$ is a positive stable matrix (where $\operatorname{Re}(z)$ denotes the real part of $z \in \mathbb{C}$ ).

2. Matrices $A_{i}, B_{i}, i=1,2$, are $m \times m$ complex matrices, and we assume that the block matrix

$$
\left(\begin{array}{ll}
A_{1} & B_{1} \\
A_{2} & B_{2}
\end{array}\right) \text { is regular, }
$$

and also that the matrix pencil

$$
A_{1}+\rho B_{1} \text { is regular. }
$$

Condition (7) is well known in the literature of singular systems of differential equations, see [17], and involves the existence of some $\rho_{0} \in \mathbb{C}$ so that matrix $A_{1}+\rho_{0} B_{1}$ is invertible. In this case, matrix $A_{1}+\rho B_{1}$ is invertible with the possible exception of at most a finite number of complex numbers $\rho$. In particular, we may assume that $\rho_{0} \in \mathbb{R}$.

Using condition (7) we can introduce the following matrices $\tilde{A}_{1}$ and $\tilde{B}_{1}$ defined by

$$
\tilde{A}_{1}=\left(A_{1}+\rho_{0} B_{1}\right)^{-1} A_{1}, \quad \tilde{B}_{1}=\left(A_{1}+\rho_{0} B_{1}\right)^{-1} B_{1}
$$

which satisfy the condition $\tilde{A}_{1}+\rho_{0} \tilde{B}_{1}=I$, where matrix $I$ denotes, as usual, the identity matrix. Under hypothesis (6), is it easy to show that matrix $B_{2}-\left(A_{2}+\rho_{0} B_{2}\right) \tilde{B}_{1}$ is regular (see [18] for details) and we can introduce matrices $\tilde{A}_{2}$ and $\tilde{B}_{2}$ defined by

$$
\tilde{A}_{2}=\left[B_{2}-\left(A_{2}+\rho_{0} B_{2}\right) \tilde{B}_{1}\right]^{-1} A_{2}, \quad \tilde{B}_{2}=\left[B_{2}-\left(A_{2}+\rho_{0} B_{2}\right) \tilde{B}_{1}\right]^{-1} B_{2}
$$

that satisfy the conditions $\tilde{B}_{2}-\left(\tilde{A}_{2}+\rho_{0} \tilde{B}_{2}\right) \tilde{B}_{1}=I, \quad \tilde{B}_{2} \tilde{A}_{1}-\tilde{A}_{2} \tilde{B}_{1}=I$.

Under the above assumptions, the homogeneous problem (1)-(4) was solved in [15] [16] in two different cases:

(a) If we consider the following hypotheses:

exist $b_{1} \in \sigma\left(\tilde{B}_{1}\right)-\{0\}, b_{2} \in \sigma\left(\tilde{B}_{2}\right)$, and $v \in \mathbb{C}^{m}-\{0\}$, such that $\left(\tilde{B}_{1}-b_{1} I\right) v=\left(\tilde{B}_{2}-b_{2} I\right) v=0$

Then, if the vector valued function $f(x)$ satisfies hypotheses

$$
\left.\begin{array}{c}
f \in \mathcal{C}^{2}([0,1]) \\
\left(1-\rho_{0} b_{1}\right) f(0)+b_{1} f^{\prime}(0)=0 \\
-\left(\frac{1-b_{2}+\rho_{0} b_{1} b_{2}}{b_{1}}\right) f(1)+b_{2} f^{\prime}(1)=0
\end{array}\right\}
$$


with the additional condition:

$$
f(x) \in \operatorname{Ker}\left(\tilde{B}_{1}-b_{1} I\right) \cap \operatorname{Ker}\left(\tilde{B}_{2}-b_{2} I\right), \quad 0 \leq x \leq 1
$$

$$
\operatorname{Ker}\left(\tilde{B}_{1}-b_{1} I\right) \cap \operatorname{Ker}\left(\tilde{B}_{2}-b_{2} I\right) \text { is an invariant subspace with respect to matrix } A \text {, }
$$

where a subspace $E$ of $\mathbb{C}^{m}$ is invariant by the matrix $A \in \mathbb{C}^{m \times m}$ if $A(E) \subset E$, we can construct an exact series solution $u(x, t)$ of homogeneous problem (1)-(4). This construction was made in Ref. [15].

(b) If we consider the following hypotheses:

$$
0 \in \sigma\left(\tilde{B}_{1}\right), a_{2} \in \sigma\left(\tilde{A}_{2}\right) \text {, and we have } w \in \mathbb{C}^{m}-\{0\} \text {, so that } \tilde{B}_{1} w=\left(\tilde{A}_{2}-a_{2} I\right) w=0
$$

Then, if the vector valued function $f(x)$ satisfies the hypotheses

$$
\left.\begin{array}{c}
f \in \mathcal{C}^{2}([0,1]) \\
f(0)=0 \\
a_{2} f(1)+f^{\prime}(1)=0
\end{array}\right\}
$$

under the additional condition:

$$
f(x) \in \operatorname{Ker}\left(\tilde{B}_{1}\right) \cap \operatorname{Ker}\left(\tilde{A}_{2}-a_{2} I\right), \quad 0 \leq x \leq 1
$$

$$
\operatorname{Ker}\left(\tilde{B}_{1}\right) \cap \operatorname{Ker}\left(\tilde{A}_{2}-a_{2} I\right) \text { is an invariant subspace respect to matrix } A \text {, }
$$

then we can construct an exact series solution $u(x, t)$ of homogeneous problem (1)-(4). This construction was made in Ref. [16].

Observe that under the different hypotheses (a) and (b), the exact solution of problem (1)-(1) is given by the series

$$
u(x, t)=\alpha\left(\left(1-\rho_{0} b_{1}\right) x-b_{1}\right) C(0)+\sum_{\lambda_{n} \in \mathcal{F}} \mathrm{e}^{-\lambda_{n}^{2} A t} X_{\lambda_{n}}(x) C\left(\lambda_{n}\right), \quad x \in[0,1], \quad t \geq 0
$$

where, under hypothesis (a), the value of $\alpha$ is given by

$$
\alpha=\left\{\begin{array}{l}
1 \text { if } \frac{\left(1-b_{2}+\rho_{0} b_{1} b_{2}\right)\left(1-\rho_{0} b_{1}\right)}{b_{1}}=1 \\
0 \text { if } \frac{\left(1-b_{2}+\rho_{0} b_{1} b_{2}\right)\left(1-\rho_{0} b_{1}\right)}{b_{1}} \neq 1
\end{array}\right.
$$

and $\mathcal{F}$ is the set of eigenvalues $\lambda_{n} \in(n \pi,(n+1) \pi)$, where $\lambda_{n}$ is the solution of the equation

$$
\lambda \cot (\lambda)=\frac{\left(1-b_{2}+\rho_{0} b_{1} b_{2}\right)\left(1-\rho_{0} b_{1}\right)}{b_{1}}-b_{1} b_{2} \lambda^{2}
$$

with an additional solution $\lambda_{0} \in(0, \pi)$ if

$$
\frac{\left(1-b_{2}+\rho_{0} b_{1} b_{2}\right)\left(1-\rho_{0} b_{1}\right)}{b_{1}}<1
$$

and under hypothesis (b), the value of $\alpha$ is given by

$$
\alpha= \begin{cases}1 & \text { if }-a_{2}=1 \\ 0 & \text { if }-a_{2} \neq 1\end{cases}
$$


and $\mathcal{F}$ is the set of eigenvalues $\lambda_{n} \in(n \pi,(n+1) \pi)$, where $\lambda_{n}$ is the solution of the equation

$$
\lambda \cot (\lambda)=-a_{2}
$$

with an additional solution $\lambda_{0} \in(0, \pi)$ if

$$
-a_{2}<1
$$

Under both hypotheses (a) and (b), the value of $X_{\lambda_{n}}(x), C\left(\lambda_{n}\right)$ and $C(0)$ are given by

$$
\begin{gathered}
X_{\lambda_{n}}(x)=\left(\left(1-\rho_{0} b_{1}\right) \sin \left(\lambda_{n} x\right)-b_{1} \lambda_{n} \cos \left(\lambda_{n} x\right)\right) \\
C\left(\lambda_{n}\right)=\frac{\int_{0}^{1}\left(\left(1-\rho_{0} b_{1}\right) \sin \left(\lambda_{n} x\right)-b_{1} \lambda_{n} \cos \left(\lambda_{n} x\right)\right) f(x) \mathrm{d} x}{\int_{0}^{1}\left(\left(1-\rho_{0} b_{1}\right) \sin \left(\lambda_{n} x\right)-b_{1} \lambda_{n} \cos \left(\lambda_{n} x\right)\right)^{2} \mathrm{~d} x}
\end{gathered}
$$

and

$$
C(0)=\frac{\int_{0}^{1}\left(\left(1-\rho_{0} b_{1}\right) x-b_{1}\right) f(x) \mathrm{d} x}{\int_{0}^{1}\left(\left(1-\rho_{0} b_{1}\right) x-b_{1}\right)^{2} \mathrm{~d} x}
$$

taking $b_{1}=0$ in Formulaes (23)-(25) if we consider hypothesis $(b)$.

The series solution of problem (1)-(4) given in (16) presents some computational difficulties:

(a) The infiniteness of the series.

(b) Eigenvalues $\lambda_{n}$ are not exactly computable because Equation (18) (or Equation (21) under hypothesis (b) holds) is not solvable in a closed form, although well known and efficient algorithms for approximation, see references [13] [19] [20].

(c) Other problem is the calculation of the matrix exponential, which may present difficulties, see [21] [22] for example.

For this reason we propose in this paper to solve the following problem:

Given an admissible error $\varepsilon>0$ and a bounded subdomain $D\left[t_{0}, t_{1}\right]=[0,1] \times\left[t_{0}, t_{1}\right], t_{0}>0$. How do we construct an approximation that avoids the above-quoted difficulties and whose error with respect to the exact solution (16) is less than $\varepsilon$ uniformly in $D\left[t_{0}, t_{1}\right]$ ?

This paper deals with the construction of analytic-numerical solutions of problem (1)-(4) in a subdomain $D\left[t_{0}, t_{1}\right]=[0,1] \times\left[t_{0}, t_{1}\right], t_{0}>0$, with a priori error $\varepsilon>0$. The work is organized as follows: in Section 2 we construct the approximate solution. In Section 3 we will introduce an algorithm and give an illustrative example.

Throughout this paper we will assume the results and nomenclature given in [15] [16]. If $B=\left(b_{i j}\right)$ is a matrix in $\mathbb{C}^{m \times m}$, its 2-norm denoted by $\|B\|$ is defined by ([23], p. 56)

$$
\|B\|=\sup _{z \neq 0} \frac{\|B z\|_{2}}{\|z\|_{2}}
$$

where for a vector $y$ in $\mathbb{C}^{m},\|z\|_{2}$ is the usual euclidean norm of $y$, and the 2-norm satisfies

$$
\max _{i, j}\left|b_{i j}\right| \leq\|B\| \leq m \max _{i, j}\left|b_{i j}\right|
$$

Let us introduce the notation

$$
\alpha(C)=\max \{\operatorname{Re}(z) ; z \in \sigma(C)\}
$$

and by ([23], p. 556) it follows that

$$
\left\|\mathrm{e}^{t B}\right\| \leq \mathrm{e}^{\alpha(B) t} \sum_{k=0}^{m-1} \frac{\|\sqrt{m} B\|^{k} t^{k}}{k !}
$$




\section{The Proposed Approximation}

Let $(x, t) \in D\left[t_{0}, t_{1}\right]=[0,1] \times\left[t_{0}, t_{1}\right], t_{0}>0$, be and we take an admissible error $\varepsilon>0$. Observe first that given (24), using Parseval's identity for scalar Sturm-Liouville problems, see [24] and ([11], p. 223), one gets that

$$
\left\|C\left(\lambda_{n}\right)\right\|^{2} \leq \int_{0}^{1}\|f(x)\|^{2} \mathrm{~d} x, \quad \lambda_{n} \in \mathcal{F}
$$

Thus, we can take a positive constant $M>0$, defined by

$$
M=\int_{0}^{1}\|f(x)\|^{2} \mathrm{~d} x
$$

satisfying

$$
\left\|C\left(\lambda_{n}\right)\right\|^{2} \leq M, \quad \lambda_{n} \in \mathcal{F}
$$

Moreover, by (23), we have

$$
\left|X_{\lambda_{n}}(x)\right|^{2}=\left|\left(\left(1-\rho_{0} b_{1}\right) \sin \left(\lambda_{n} x\right)-b_{1} \lambda_{n} \cos \left(\lambda_{n} x\right)\right)\right|^{2} \leq\left|1-\rho_{0} b_{1}\right|^{2}+\left|b_{1}\right|^{2} \lambda_{n}^{2}+2\left|1-\rho_{0} b_{1}\right|\left|b_{1}\right| \lambda_{n} .
$$

If we define $\beta>0$ by

$$
\beta=\max \left\{\left|1-\rho_{0} b_{1}\right|^{2},\left|b_{1}\right|^{2},\left|1-\rho_{0} b_{1}\right|\left|b_{1}\right|\right\}
$$

we have that

$$
\left|X_{\lambda_{n}}(x)\right|^{2} \leq \beta\left(1+\lambda_{n}\right)^{2}, \quad \lambda_{n} \in \mathcal{F}
$$

On the other hand, we know from (27) that

$$
\left\|\mathrm{e}^{-A \lambda_{n}^{2} t}\right\| \leq \mathrm{e}^{-\alpha(A) \lambda_{n}^{2} t} \sum_{k=0}^{m-1} \frac{\|\sqrt{m} A\|^{k} t^{k} \lambda_{n}^{2 k}}{k !}
$$

where, as $\lambda_{n} \geq 1, \quad n \geq 1$, we have for $t \in\left[t_{0}, t_{1}\right]$ :

$$
\left\|\mathrm{e}^{-A \lambda_{n}^{2} t}\right\|^{2} \leq \mathrm{e}^{-2 \alpha(A) \lambda_{n}^{2} t_{0}}\left(\sum_{k=0}^{m-1} \frac{\|\sqrt{m} A\|^{k} t_{1}^{k}}{k !}\right)^{2} \lambda_{n}^{4 m-4}=L^{2} \lambda_{n}^{4 m-4} \mathrm{e}^{-2 \alpha(A) \lambda_{n}^{2} t_{0}}=L^{2} \lambda_{n}^{-4}\left(\lambda_{n}^{4 m} \mathrm{e}^{-2 \alpha(A) \lambda_{n}^{2} t_{0}}\right)
$$

where

$$
L=\sum_{k=0}^{m-1} \frac{\|\sqrt{m} A\|^{k} t_{1}^{k}}{k !}>0
$$

Observe that for a fixed $m \geq 0$ the numerical series $\sum_{\lambda_{n} \in \mathcal{F}} \lambda_{n}^{4 m} \mathrm{e}^{-2 \alpha(A) \lambda_{n}^{2} t_{0}}$ is convergent, because using Lemma 1 of Ref. [15] if hypothesis (a) holds, or Lemma 2 of Ref. [16] if hypothesis (b) holds, one gets $\lim _{n \rightarrow \infty} \lambda_{n}=\infty, \lim _{n \rightarrow \infty}\left(\lambda_{n+1}-\lambda_{n}\right)=\pi$, and by application of D'Alembert's criterion for series:

$$
\lim _{n \rightarrow \infty} \frac{a_{n+1}}{a_{n}}=\lim _{n \rightarrow \infty}\left(\frac{\lambda_{n+1}}{\lambda_{n}}\right)^{4 m} \mathrm{e}^{-2 \alpha(A) t_{0}\left(\lambda_{n+1}^{2}-\lambda_{n}^{2}\right)} \leq \lim _{n \rightarrow \infty} \mathrm{e}^{-\alpha(A) t_{0}\left(\lambda_{n+1}^{2}-\lambda_{n}^{2}\right)}\left(\frac{n+2}{n}\right)^{4 m}=\mathrm{e}^{\lim _{n \rightarrow \infty}-\alpha(A) t_{0} \pi\left(\lambda_{n+1}+\lambda_{n}\right)}=0
$$

then

$$
\lim _{n \rightarrow \infty} \lambda_{n}^{4 m} \mathrm{e}^{-2 \alpha(A) \lambda_{n}^{2} t_{0}}=0 .
$$

Taking into account that $\left(1+\lambda_{n}\right)^{2}>1$ and $M>0, \quad \beta>0$, it follows that 


$$
\frac{1}{M \beta\left(1+\lambda_{n}\right)^{2} L^{2}}<\frac{1}{M \beta L^{2}}<1
$$

and by (34) there is a positive integer $n_{0}$ so that

$$
\lambda_{n}^{4 m} \mathrm{e}^{-2 \alpha(A) \lambda_{n}^{2} t_{0}}<\frac{1}{M \beta\left(1+\lambda_{n}\right)^{2} L^{2}}, \quad \forall n \geq n_{0}
$$

Using (29), (31), (32) and (36), if $n \geq n_{0}$, we have

$$
\left\|\mathrm{e}^{-A \lambda_{n}^{2} t} X_{\lambda_{n}}(x) C\left(\lambda_{n}\right)\right\|^{2} \leq\left\|\mathrm{e}^{-A \lambda_{n}^{2} t}\right\|^{2}\left|X_{\lambda_{n}}(x)\right|^{2}\left\|C\left(\lambda_{n}\right)\right\|^{2}=M \beta\left(1+\lambda_{n}\right)^{2} L^{2} \lambda_{n}^{-4}\left(\lambda_{n}^{4 m} \mathrm{e}^{-2 \alpha(A) \lambda_{n}^{2} t_{0}}\right) \leq \lambda_{n}^{-4}
$$

As eigenvalues $\lambda_{n} \in(n \pi,(n+1) \pi)$, then, for $n>1$ it follows that

$$
\frac{1}{\lambda_{n}^{4}}<\frac{1}{n^{4}}
$$

Taking into account that $\sum_{n \geq 1} \frac{1}{n^{4}}=\frac{\pi^{4}}{90}$, from (37) one gets that

$$
\begin{gathered}
\left\|\sum_{\substack{\lambda_{n} \in \mathcal{F} \\
n \geq n_{0}}} \mathrm{e}^{-A \lambda_{n}^{2} t} X_{\lambda_{n}}(x) C\left(\lambda_{n}\right)\right\|^{2} \leq \sum_{\substack{\lambda_{n} \in \mathcal{F} \\
n \geq n_{0}}}\left\|\mathrm{e}^{-A \lambda_{n}^{2} t} X_{\lambda_{n}}(x) C\left(\lambda_{n}\right)\right\|^{2} \\
\leq \sum_{\substack{\lambda_{n} \in \mathcal{F} \\
n \geq n_{0}}} \frac{1}{\lambda_{n}^{4}} \\
\leq \sum_{n \geq n_{0}} \frac{1}{n^{4}} \\
=\frac{\pi^{4}}{90}-\sum_{n=1}^{n_{0}} \frac{1}{n^{4}}
\end{gathered}
$$

We take the first positive integer $n_{1}$ so that

$$
\sum_{n=1}^{n_{1}} \frac{1}{n^{4}} \geq \frac{\pi^{4}}{90}-\frac{\varepsilon}{3}, \quad n_{1} \geq n_{0}
$$

We define the vector valued function $u\left(x, t, n_{1}\right)$ as

$$
u\left(x, t, n_{1}\right)=\alpha\left(\left(1-\rho_{0} b_{1}\right) x-b_{1}\right) C(0)+\sum_{\substack{\lambda_{n} \in \mathcal{F} \\ n \leq n_{1}}} \mathrm{e}^{-\lambda_{n}^{2} A t} X_{\lambda_{n}}(x) C\left(\lambda_{n}\right), \quad(x, t) \in D\left[t_{0}, t_{1}\right]
$$

Using (38) one gets that

$$
\begin{aligned}
\left\|u(x, t)-u\left(x, t, n_{1}\right)\right\|^{2} & \leq\left\|\sum_{\substack{\lambda_{n} \in \mathcal{F} \\
n \geq n_{1}}} \mathrm{e}^{-\lambda_{n}^{2} A t} X_{\lambda_{n}}(x) C\left(\lambda_{n}\right)\right\|^{2} \\
& \leq \frac{\pi^{4}}{90}-\sum_{n=1}^{n_{1}} \frac{1}{n^{4}} \\
& \leq \frac{\pi^{4}}{90}-\frac{\pi^{4}}{90}+\frac{\varepsilon}{3} \\
& =\frac{\varepsilon}{3},
\end{aligned}
$$


thus

$$
\left\|u(x, t)-u\left(x, t, n_{1}\right)\right\|^{2} \leq \frac{\varepsilon}{3}, \quad(x, t) \in D\left[t_{0}, t_{1}\right]
$$

Remark 1. Note that to determine the positive integer $n_{0}$ we need to check condition (36), which requires knowledge the exact eigenvalues $\lambda_{n}$. From Ref. [15] [16] it is well know that $\lambda_{n} \in(n \pi,(n+1) \pi)$, then

$$
\lambda_{n}^{4 m} \mathrm{e}^{-2 \alpha(A) \lambda_{n}^{2} t_{0}}<(n \pi)^{4 m} \mathrm{e}^{-2 \alpha(A) n^{2} \pi^{2} t_{0}}
$$

and by (35), we can replace condition (36) by take the first positive integer $n_{0}$ satisfying

$$
(n \pi)^{4 m} \mathrm{e}^{-2 \alpha(A) n^{2} \pi^{2} t_{0}}<\frac{1}{M \beta L^{2}} .
$$

Approximation $u\left(x, t, n_{1}\right)$ defined by (41) involves computation of the exact eigenvalues $\lambda_{n}, n \leq n_{1}$ which is not easy in practice. Now we study the admissible tolerance when one considers approximate eigenvalues $\widetilde{\lambda}_{n}, n \leq n_{1}$ in expression (41), taking

$$
\tilde{u}\left(x, t, n_{1}\right)=\alpha\left(\left(1-\rho_{0} b_{1}\right) x-b_{1}\right) C(0)+\sum_{n \leq n_{1}} \mathrm{e}^{-{\widetilde{\lambda_{n}}}^{2} A t} X_{\widetilde{\lambda_{n}}}(x) C\left(\widetilde{\lambda_{n}}\right)
$$

where

$$
\begin{array}{r}
X_{\widetilde{\lambda}_{n}}(x)=\left(\left(1-\rho_{0} b_{1}\right) \sin \left(\widetilde{\lambda}_{n} x\right)-b_{1} \widetilde{\lambda}_{n} \cos \left(\widetilde{\lambda}_{n} x\right)\right), \quad x \in[0,1] \\
C\left(\widetilde{\lambda}_{n}\right)=\frac{\int_{0}^{1}\left(\left(1-\rho_{0} b_{1}\right) \sin \left(\widetilde{\lambda}_{n} x\right)-b_{1} \widetilde{\lambda}_{n} \cos \left(\widetilde{\lambda}_{n} x\right)\right) f(x) \mathrm{d} x}{\int_{0}^{1}\left(\left(1-\rho_{0} b_{1}\right) \sin \left(\widetilde{\lambda}_{n} x\right)-b_{1} \widetilde{\lambda}_{n} \cos \left(\widetilde{\lambda}_{n} x\right)\right)^{2} \mathrm{~d} x}
\end{array}
$$

with $C(0)$ defined by (25). Note that

$$
\begin{aligned}
\mathrm{e}^{-\widetilde{\lambda}_{n}^{2} A t} & X_{\widetilde{\lambda}_{n}}(x) C\left(\widetilde{\lambda}_{n}\right)-\mathrm{e}^{-\lambda_{n}^{2} A t} X_{\lambda_{n}}(x) C\left(\lambda_{n}\right) \\
= & \left(\mathrm{e}^{-\widetilde{\lambda}_{n}^{2} A t}-\mathrm{e}^{-\lambda_{n}^{2} A t}\right)\left\{\left(1-\rho_{0} b_{1}\right) \sin \left(\widetilde{\lambda}_{n} x\right)-b_{1} \widetilde{\lambda}_{n} \cos \left(\widetilde{\lambda}_{n} x\right)\right\} C\left(\widetilde{\lambda_{n}}\right) \\
& +\mathrm{e}^{-\lambda_{n}^{2} A t}\left\{\left(1-\rho_{0} b_{1}\right) \sin \left(\widetilde{\lambda}_{n} x\right)-b_{1} \widetilde{\lambda}_{n} \cos \left(\widetilde{\lambda}_{n} x\right)-\left(1-\rho_{0} b_{1}\right) \sin \left(\lambda_{n} x\right)+b_{1} \lambda_{n} \cos \left(\lambda_{n} x\right)\right\} C\left(\widetilde{\lambda}_{n}\right) \\
& +\mathrm{e}^{-\lambda_{n}^{2} A t}\left\{\left(1-\rho_{0} b_{1}\right) \sin \left(\lambda_{n} x\right)-b_{1} \lambda_{n} \cos \left(\lambda_{n} x\right)\right\}\left(C\left(\widetilde{\lambda}_{n}\right)-C\left(\lambda_{n}\right)\right) .
\end{aligned}
$$

It is easy to see that

$$
\begin{aligned}
& \left|\left(1-\rho_{0} b_{1}\right) \sin \left(\widetilde{\lambda}_{n} x\right)-b_{1} \widetilde{\lambda}_{n} \cos \left(\widetilde{\lambda}_{n} x\right)\right| \leq\left|1-\rho_{0} b_{1}\right|+\left|b_{1}\right| \widetilde{\lambda}_{n}, \\
& \left|\left(1-\rho_{0} b_{1}\right) \sin \left(\lambda_{n} x\right)-b_{1} \lambda_{n} \cos \left(\lambda_{n} x\right)\right| \leq\left|1-\rho_{0} b_{1}\right|+\left|b_{1}\right| \lambda_{n},
\end{aligned}
$$

and

$$
\begin{aligned}
& \left|\left(1-\rho_{0} b_{1}\right) \sin \left(\widetilde{\lambda}_{n} x\right)-b_{1} \widetilde{\lambda}_{n} \cos \left(\widetilde{\lambda}_{n} x\right)-\left(1-\rho_{0} b_{1}\right) \sin \left(\lambda_{n} x\right)+b_{1} \lambda_{n} \cos \left(\lambda_{n} x\right)\right| \\
& \leq\left(\left|1-\rho_{0} b_{1}\right|+\left|b_{1}\right|\left(1+\lambda_{n}\right)\right)\left|\lambda_{n}-\widetilde{\lambda}_{n}\right| .
\end{aligned}
$$

Replacing in (47) and taking norms, one gets

$$
\begin{aligned}
\left\|\mathrm{e}^{-{\widetilde{\lambda_{n}}}^{2} A t} X_{\widetilde{\lambda}_{n}}(x) C\left(\widetilde{\lambda_{n}}\right)-\mathrm{e}^{-\lambda_{n}^{2} A t} X_{\lambda_{n}}(x) C\left(\lambda_{n}\right)\right\| & \leq \mid \mathrm{e}^{-\widetilde{\lambda}_{n}^{2} A t}-\mathrm{e}^{-\lambda_{n}^{2} A t}\left\|\left(\left|1-\rho_{0} b_{1}\right|+\left|b_{1}\right| \widetilde{\lambda}_{n}\right)\right\| C\left(\widetilde{\lambda_{n}}\right) \| \\
& +\left\|\mathrm{e}^{-\lambda_{n}^{2} A t}\right\|\left(\left(\left|1-\rho_{0} b_{1}\right|+\left|b_{1}\right|\left(1+\lambda_{n}\right)\right)\left|\lambda_{n}-\widetilde{\lambda_{n}}\right|\right)\left\|C\left(\widetilde{\lambda_{n}}\right)\right\| \\
& +\left\|\mathrm{e}^{-\lambda_{n}^{2} A t}\right\|\left(\left|1-\rho_{0} b_{1}\right|+\left|b_{1}\right| \lambda_{n}\right)\left\|C\left(\widetilde{\lambda_{n}}\right)-C\left(\lambda_{n}\right)\right\| .
\end{aligned}
$$


E. Defer et al.

We define $I(\rho)$ for $\rho>0$ by

$$
I(\rho)=\int_{0}^{1}\left(\left(1-\rho_{0} b_{1}\right) \sin (\rho x)-b_{1} \rho \cos (\rho x)\right)^{2} \mathrm{~d} x
$$

by applying the Cauchy-Schwarz inequality for integrals and (28), one gets:

$$
\int_{0}^{1}\|f(x)\| \mathrm{d} x \leq\left(\int_{0}^{1}\|f(x)\|^{2} \mathrm{~d} x\right)^{\frac{1}{2}}=\sqrt{M}
$$

We have

$$
\begin{aligned}
\left\|C\left(\widetilde{\lambda_{n}}\right)\right\| & \leq \frac{1}{I\left(\widetilde{\lambda}_{n}\right)} \int_{0}^{1}\left|\left(1-\rho_{0} b_{1}\right) \sin \left(\widetilde{\lambda}_{n} x\right)-b_{1} \widetilde{\lambda}_{n} \cos \left(\widetilde{\lambda}_{n} x\right)\right|\|f(x)\| \mathrm{d} x \\
& \leq \frac{1}{I\left(\widetilde{\lambda_{n}}\right)} \int_{0}^{1}\left(\left|1-\rho_{0} b_{1}\right|+\left|b_{1}\right| \widetilde{\lambda}_{n}\right)\|f(x)\| \mathrm{d} x \\
& \leq \frac{\left|1-\rho_{0} b_{1}\right|+\left|b_{1}\right| \widetilde{\lambda}_{n}}{I\left(\widetilde{\lambda}_{n}\right)} \int_{0}^{1}\|f(x)\| \mathrm{d} x \\
& \leq \frac{\left|1-\rho_{0} b_{1}\right|+\left|b_{1}\right| \tilde{\lambda}_{n}}{I\left(\widetilde{\lambda_{n}}\right)} \sqrt{M} .
\end{aligned}
$$

Taking $\gamma>0$ satisfying

$$
\min _{n \leq n_{1}}\left\{I(\rho), \rho=\lambda_{n}, \rho=\widetilde{\lambda}_{n}\right\} \geq 1 / \gamma
$$

it follows that

$$
\left\|C\left(\widetilde{\lambda}_{n}\right)\right\| \leq \gamma\left(\left|1-\rho_{0} b_{1}\right|+\left|b_{1}\right| \widetilde{\lambda}_{n}\right) \sqrt{M}
$$

Moreover, working component by component:

$$
\begin{gathered}
C\left(\tilde{\lambda}_{n}\right)_{i}-C\left(\lambda_{n}\right)_{i} \\
=\frac{\int_{0}^{1} X_{\widetilde{\lambda_{n}}}(x) f_{i}(x) \mathrm{d} x}{I\left(\widetilde{\lambda_{n}}\right)}-\frac{\int_{0}^{1} X_{\lambda_{n}}(x) f_{i}(x) \mathrm{d} x}{I\left(\lambda_{n}\right)} \\
=\frac{I\left(\lambda_{n}\right) \int_{0}^{1} X_{\widetilde{\lambda_{n}}}(x) f_{i}(x) \mathrm{d} x-I\left(\widetilde{\lambda_{n}}\right) \int_{0}^{1} X_{\lambda_{n}}(x) f_{i}(x) \mathrm{d} x}{I\left(\widetilde{\lambda_{n}}\right) I\left(\lambda_{n}\right)} \\
=\frac{\left(I\left(\lambda_{n}\right)-I\left(\widetilde{\lambda_{n}}\right)\right) \int_{0}^{1} X_{\widetilde{\lambda_{n}}}(x) f_{i}(x) \mathrm{d} x-I\left(\widetilde{\lambda}_{n}\right) \int_{0}^{1}\left(X_{\lambda_{n}}(x)-X_{\widetilde{\lambda_{n}}}(x)\right) f_{i}(x) \mathrm{d} x}{I\left(\widetilde{\lambda_{n}}\right) I\left(\lambda_{n}\right)}
\end{gathered}
$$

Applying the Cauchy-Schwarz inequality for integrals again:

$$
\int_{0}^{1}\left|X_{\widetilde{\lambda_{n}}}(x) f_{i}(x)\right| \mathrm{d} x \leq\left(\int_{0}^{1}\left|f_{i}(x)\right|^{2} \mathrm{~d} x\right)^{\frac{1}{2}}\left(\int_{0}^{1}\left|X_{\widetilde{\lambda_{n}}}(x)\right|^{2} \mathrm{~d} x\right)^{\frac{1}{2}}=\left(\int_{0}^{1}\left|f_{i}(x)\right|^{2} \mathrm{~d} x\right)^{\frac{1}{2}}\left(I\left(\widetilde{\lambda_{n}}\right)\right)^{\frac{1}{2}}
$$

and

$$
\begin{gathered}
\int_{0}^{1}\left|\left(X_{\lambda_{n}}(x)-X_{\widetilde{\lambda_{n}}}(x)\right) f_{i}(x)\right| \mathrm{d} x \\
\leq\left(\left|1-\rho_{0} b_{1}\right|+\left|b_{1}\right|\left(1+\lambda_{n}\right)\right)\left|\lambda_{n}-\widetilde{\lambda_{n}}\right| \int_{0}^{1}\left|f_{i}(x)\right| \mathrm{d} x
\end{gathered}
$$

8 


$$
\leq\left(\left|1-\rho_{0} b_{1}\right|+\left|b_{1}\right|\left(1+\lambda_{n}\right)\right)\left|\lambda_{n}-\widetilde{\lambda}_{n}\right|\left(\int_{0}^{1}\left|f_{i}(x)\right|^{2} \mathrm{~d} x\right)^{\frac{1}{2}}
$$

By (55) and taking into account (57) and (58):

$$
\begin{aligned}
& \left|C\left(\widetilde{\lambda}_{n}\right)_{i}-C\left(\lambda_{n}\right)_{i}\right| \\
& \leq \frac{1}{I\left(\widetilde{\lambda_{n}}\right) I\left(\lambda_{n}\right)}\left(\left|I\left(\lambda_{n}\right)-I\left(\widetilde{\lambda_{n}}\right)\right| \int_{0}^{1}\left|X_{\widetilde{\lambda_{n}}}(x) f_{i}(x)\right| \mathrm{d} x+I\left(\widetilde{\lambda_{n}}\right) \int_{0}^{1}\left|\left(X_{\lambda_{n}}(x)-X_{\widetilde{\lambda}_{n}}(x)\right) f_{i}(x)\right| \mathrm{d} x\right) \\
& =\frac{\left(\int_{0}^{1}\left|f_{i}(x)\right|^{2} \mathrm{~d} x\right)^{\frac{1}{2}}}{I\left(\lambda_{n}\right)}\left(\frac{\left|I\left(\lambda_{n}\right)-I\left(\widetilde{\lambda_{n}}\right)\right|}{\left(I\left(\widetilde{\lambda_{n}}\right)\right)^{\frac{1}{2}}}+\left|I\left(\lambda_{n}\right)-I\left(\widetilde{\lambda_{n}}\right)\right|\left(\left|1-\rho_{0} b_{1}\right|+\left|b_{1}\right|\left(1+\lambda_{n}\right)\right)\right) .
\end{aligned}
$$

Note that from the definition of $I(\rho)$, (52), it follows that

$$
\left|I\left(\lambda_{n}\right)-I\left(\widetilde{\lambda}_{n}\right)\right| \leq\left(\left|1-\rho_{0} b_{1}\right|+\left|b_{1}\right|\left(1+\widetilde{\lambda}_{n}\right)\right)\left(2\left|1-\rho_{0} b_{1}\right|+\left|b_{1}\right|\left(\widetilde{\lambda}_{n}+\lambda_{n}\right)\right)\left|\widetilde{\lambda}_{n}-\lambda_{n}\right|
$$

then, replacing in (60) one gets

$$
\begin{aligned}
\left|C\left(\widetilde{\lambda}_{n}\right)_{i}-C\left(\lambda_{n}\right)_{i}\right| \leq & \frac{\left(\int_{0}^{1}\left|f_{i}(x)\right|^{2} \mathrm{~d} x\right)^{\frac{1}{2}}}{I\left(\lambda_{n}\right)}\left\{\left(\left|1-\rho_{0} b_{1}\right|+\left|b_{1}\right|\left(1+\lambda_{n}\right)\right)\right. \\
& \left.+\left(\left|1-\rho_{0} b_{1}\right|+\left|b_{1}\right|\left(1+\widetilde{\lambda}_{n}\right)\right)\left(2\left|1-\rho_{0} b_{1}\right|+\left|b_{1}\right|\left(\widetilde{\lambda}_{n}+\lambda_{n}\right)\right)\left(I\left(\widetilde{\lambda}_{n}\right)\right)^{-\frac{1}{2}}\right\}\left|\widetilde{\lambda}_{n}-\lambda_{n}\right| .
\end{aligned}
$$

We take

$$
\Lambda \geq \max _{n \leq n_{1}}\left\{\lambda_{n}, \widetilde{\lambda}_{n}\right\}
$$

then, if we define

$$
\mathcal{A}=\left|1-\rho_{0} b_{1}\right|+\left|b_{1}\right|(1+\Lambda), \quad \mathcal{B}=2\left|1-\rho_{0} b_{1}\right|+2\left|b_{1}\right| \Lambda
$$

from (54) we have that

$$
\left\|C\left(\widetilde{\lambda}_{n}\right)\right\| \leq \gamma \mathcal{A} \sqrt{M}
$$

and from (62) and (53):

$$
\begin{aligned}
\left|C\left(\widetilde{\lambda}_{n}\right)_{i}-C\left(\lambda_{n}\right)_{i}\right| & \leq \frac{\left(\int_{0}^{1}\left|f_{i}(x)\right|^{2} \mathrm{~d} x\right)^{\frac{1}{2}}}{I\left(\lambda_{n}\right)}\left\{\mathcal{A}+\mathcal{A B}\left(I\left(\widetilde{\lambda_{n}}\right)\right)^{-\frac{1}{2}}\right\}\left|\widetilde{\lambda}_{n}-\lambda_{n}\right| \\
& =\left(\int_{0}^{1}\left|f_{i}(x)\right|^{2} \mathrm{~d} x\right)^{\frac{1}{2}} \gamma\left\{\mathcal{A}+\mathcal{A B} \gamma^{\frac{1}{2}}\right\}\left|\widetilde{\lambda}_{n}-\lambda_{n}\right| \\
& \leq \sqrt{M} \gamma\left\{\mathcal{A}+\mathcal{A B} \gamma^{\frac{1}{2}}\right\}\left|\widetilde{\lambda}_{n}-\lambda_{n}\right| .
\end{aligned}
$$

Using the 2-norm properties, from (66) we have

$$
\left\|C\left(\tilde{\lambda}_{n}\right)-C\left(\lambda_{n}\right)\right\| \leq\left(\int_{0}^{1}\left|f_{i}(x)\right|^{2} \mathrm{~d} x\right)^{\frac{1}{2}} \gamma\left\{\mathcal{A}+\mathcal{A B} \gamma^{\frac{1}{2}}\right\}\left|\widetilde{\lambda}_{n}-\lambda_{n}\right|
$$

By other hand, we can write 


$$
\mathrm{e}^{-{\widetilde{\lambda_{n}}}^{2} A t}-\mathrm{e}^{-\lambda_{n}^{2} A t}=\mathrm{e}^{-{\widetilde{\lambda_{n}}}^{2} A t}\left(\mathrm{e}^{\left(\lambda_{n}^{2}-{\widetilde{\lambda_{n}}}^{2}\right) A t}-I\right)
$$

where taking norm, applying (32) and (33) together the mean value theorem, under the hypothesis $\left|\widetilde{\lambda}_{n}-\lambda_{n}\right|<1$, one gets

$$
\begin{aligned}
\left\|\mathrm{e}^{-\widetilde{\lambda_{n}^{2}} A t}-\mathrm{e}^{-\lambda_{n}^{2} A t}\right\| & \leq\left\|\mathrm{e}^{-\widetilde{\lambda_{n}^{2}} A t}\right\|\left(\mathrm{e}^{\left(\lambda_{n}^{2}-\widetilde{\lambda_{n}^{2}}\right)\|A\|_{1}}-1\right) \\
& \leq \mathrm{e}^{-t_{0} \alpha(A) \Lambda^{2}}\left(\sum_{k=0}^{m-1} \frac{\left(\sqrt{m} \Lambda^{2}\|A\| t_{1}\right)^{k}}{k !}\right)\left(\mathrm{e}^{\left(\lambda_{n}^{2}-\widetilde{\lambda_{n}^{2}}\right)\|A\|_{1}}-1\right) \\
& \leq \mathrm{e}^{-t_{0} \alpha(A) \Lambda^{2}} L^{\star} t_{1}\|A\| 4 \Lambda \mathrm{e}^{t_{1}\|A\| 2 \Lambda}\left|\widetilde{\lambda_{n}}-\lambda_{n}\right| .
\end{aligned}
$$

where

$$
L^{\star}=\sum_{k=0}^{m-1} \frac{\left(\sqrt{m} \Lambda^{2}\|A\| t_{1}\right)^{k}}{k !}>0
$$

Replacing in (51) we obtain

$$
\begin{aligned}
&\left\|\mathrm{e}^{-\widetilde{\lambda_{n}^{2}} A t} X_{\widetilde{\lambda_{n}}}(x) C\left(\widetilde{\lambda_{n}}\right)-\mathrm{e}^{-\lambda_{n}^{2} A t} X_{\lambda_{n}}(x) C\left(\lambda_{n}\right)\right\| \leq \mathrm{e}^{-t_{0} \alpha(A) \Lambda^{2}} L^{\star} t_{1}|| A|| 4 \Lambda \mathrm{e}^{t_{1}|| A \| 2 \Lambda} \gamma \sqrt{M} \mathcal{A}^{2}\left|\widetilde{\lambda}_{n}-\lambda_{n}\right| \\
&+\mathrm{e}^{-t_{0} \alpha(A) \Lambda^{2}} L \gamma \sqrt{M} \mathcal{A}^{2}\left|\widetilde{\lambda_{n}}-\lambda_{n}\right| \\
&+\mathrm{e}^{-t_{0} \alpha(A) \Lambda^{2}} L \mathcal{A} \sqrt{M} \gamma\left\{\mathcal{A}+\mathcal{A} \mathcal{B} \gamma^{\frac{1}{2}}\right\}\left|\widetilde{\lambda}_{n}-\lambda_{n}\right| \\
&=S\left|\widetilde{\lambda_{n}}-\lambda_{n}\right|,
\end{aligned}
$$

where

$$
S=\mathcal{A}^{2} \gamma \sqrt{M} \mathrm{e}^{-t_{0} \alpha(A) \Lambda^{2}}\left(L+L(1+\mathcal{B} \sqrt{\gamma})+L^{\star} 4 t_{1}\|A\| \Lambda \mathrm{e}^{2 t_{1}\|A\| \Lambda}\right)
$$

Given $\varepsilon>0$ and $n_{1}$, consider approximations $\tilde{\lambda}_{n}$ of $\lambda_{n}$ for $n \leq n_{1}$ satisfiying

$$
\left|\widetilde{\lambda}_{n}-\lambda_{n}\right|<\min _{n \leq n_{1}}\left\{1, \frac{\sqrt{\varepsilon}}{\sqrt{3} n_{1} S}\right\}
$$

then

$$
\begin{aligned}
\left\|u\left(x, t, n_{1}\right)-\tilde{u}\left(x, t, n_{1}\right)\right\| & =\left\|\sum_{n \leq n_{1}}\left(\mathrm{e}^{-\widetilde{\lambda_{n}^{2}} A t} X_{\widetilde{\lambda_{n}}}(x) C\left(\widetilde{\lambda_{n}}\right)-\mathrm{e}^{-\lambda_{n}^{2} A t} X_{\lambda_{n}}(x) C\left(\lambda_{n}\right)\right)\right\| \\
& \leq \sum_{n \leq n_{1}}\left\|\mathrm{e}^{-\widetilde{\lambda_{n}^{2}} A t} X_{\widetilde{\lambda_{n}}}(x) C\left(\widetilde{\lambda_{n}}\right)-\mathrm{e}^{-\lambda_{n}^{2} A t} X_{\lambda_{n}}(x) C\left(\lambda_{n}\right)\right\| \\
& \leq \sum_{n \leq n_{1}} S\left|\widetilde{\lambda_{n}}-\lambda_{n}\right| \\
& <S n_{1} \frac{\sqrt{\varepsilon}}{\sqrt{3} n_{1} S} \\
& =\frac{\sqrt{\varepsilon}}{\sqrt{3}},
\end{aligned}
$$


and therefore

$$
\left\|u\left(x, t, n_{1}\right)-\tilde{u}\left(x, t, n_{1}\right)\right\|^{2} \leq \frac{\varepsilon}{3}, \quad(x, t) \in D\left[t_{0}, t_{1}\right] .
$$

Remark 2. From (61), and taking into account the definition of $\mathcal{A}$ and $\mathcal{B}$ given in (64), it follows that

$$
\left|I\left(\lambda_{n}\right)-I\left(\widetilde{\lambda}_{n}\right)\right| \leq \mathcal{A B}\left|\widetilde{\lambda}_{n}-\lambda_{n}\right|
$$

so that, if $\left|\widetilde{\lambda_{n}}-\lambda_{n}\right|$ is enough small, it can take $I\left(\lambda_{n}\right) \approx I\left(\widetilde{\lambda_{n}}\right)$ in the computation of $\gamma$.

Similarly, can be taken in practice

$$
\Lambda \geq \max _{1 \leq n \leq n_{1}}\left\{\widetilde{\lambda}_{n}\right\}
$$

instead of the definition (63).

Approximation $\tilde{u}\left(x, t, n_{1}\right)$ need to compute the exact value of the matrix exponential $\mathrm{e}^{-\widetilde{\lambda}_{n}^{2} A t}$. However, the approximate calculation of the exponential matrix $\mathrm{e}^{-{\widetilde{\lambda_{n}}}^{2} A t}$ can be performed by methods such as those based on the Taylor series, [25] [26], based on Hermite matrix polynomials, [27], and other existing methods in the literature, see [22] [23] for example. Suppose we take the matrix $\operatorname{App}\left(\mathrm{e}^{-{\widetilde{\lambda_{n}}}^{2} A t}\right)$ as an approximation of matrix $\mathrm{e}^{-{\widetilde{\lambda_{n}}}^{2} A t}$, so that

$$
\left\|\mathrm{e}^{-{\widetilde{\lambda_{n}}}^{2} A t}-\operatorname{App}\left(\mathrm{e}^{-{\widetilde{\lambda_{n}}}^{2} A t}\right)\right\| \leq \varepsilon_{n} t_{1}, \quad t \in\left[t_{0}, t_{1}\right], \quad \varepsilon_{n}>0, \quad n \leq n_{1}
$$

We define the approximation $\mathcal{U}\left(x, t, n_{1}\right)$ by:

$$
\mathcal{U}\left(x, t, n_{1}\right)=\alpha\left(\left(1-\rho_{0} b_{1}\right) x-b_{1}\right) C(0)+\sum_{n \leq n_{1}} \operatorname{App}\left(\mathrm{e}^{-{\widetilde{\lambda_{n}}}^{2} A t}\right) X_{{\widetilde{n_{n}}}_{n}}(x) C\left(\widetilde{\lambda}_{n}\right)
$$

and from (65), (64) and (45) one gets that

$$
\begin{aligned}
\left\|\tilde{u}\left(x, t, n_{1}\right)-\mathcal{U}\left(x, t, n_{1}\right)\right\| & \leq\left\|\sum_{n \leq n_{1}}\left(\mathrm{e}^{-{\widetilde{\lambda_{n}}}^{2} A t}-\operatorname{App}\left(\mathrm{e}^{-{\widetilde{\lambda_{n}}}^{2} A t}\right)\right)\right\| \mid X_{\widetilde{\lambda_{n}}}(x)\left\|C\left(\widetilde{\lambda_{n}}\right)\right\| \\
& \leq \sum_{n \leq n_{1}}\left\|\mathrm{e}^{-{\widetilde{\lambda_{n}}}^{2} A t}-\operatorname{App}\left(\mathrm{e}^{-{\widetilde{\lambda_{n}}}^{2} A t}\right)\right\| \gamma \mathcal{A}^{2} \sqrt{M} \\
& \leq \gamma \mathcal{A}^{2} \sqrt{M} t_{1} \sum_{n \leq n_{1}} \varepsilon_{n} .
\end{aligned}
$$

We take

$$
\mathcal{K}=\max _{1 \leq n \leq n_{1}}\left\{\varepsilon_{n}\right\}
$$

and suppose we make the approximation accurate enough satisfying condition

$$
\mathcal{K}<\frac{\sqrt{\varepsilon}}{\sqrt{3} n_{1} t_{1} \gamma \mathcal{A}^{2} \sqrt{M}}
$$

Thus, if $\mathcal{K}$ satisfies (77) it follows that

$$
\left\|\tilde{u}\left(x, t, n_{1}\right)-\mathcal{U}\left(x, t, n_{1}\right)\right\|^{2} \leq \frac{\varepsilon}{3},
$$

and from (42), (72) and (78): 


$$
\begin{aligned}
\left\|u(x, t)-\mathcal{U}\left(x, t, n_{1}\right)\right\|^{2} & =\left\|u(x, t)-u\left(x, t, n_{1}\right)+u\left(x, t, n_{1}\right)-\tilde{u}\left(x, t, n_{1}\right)+\tilde{u}\left(x, t, n_{1}\right)-\mathcal{U}\left(x, t, n_{1}\right)\right\|^{2} \\
& \leq\left\|u(x, t)-u\left(x, t, n_{1}\right)\right\|^{2}+\left\|u\left(x, t, n_{1}\right)-\tilde{u}\left(x, t, n_{1}\right)\right\|^{2}+\left\|\tilde{u}\left(x, t, n_{1}\right)-\mathcal{U}\left(x, t, n_{1}\right)\right\|^{2} \\
& \leq \frac{\varepsilon}{3}+\frac{\varepsilon}{3}+\frac{\varepsilon}{3} \\
& =\varepsilon .
\end{aligned}
$$

Summarizing, the following results has been established:

Theorem 1. We consider problem (1)-(4) satisfying hypotheses (5), (6) and (7). Let $\varepsilon>0$, $D\left[t_{0}, t_{1}\right]=[0,1] \times\left[t_{0}, t_{1}\right]$. Suppose that the hypothesis (a) is verified, this ensures that there is an exact solution $u(x, t)$ of problem (1)-(4), see Ref. [15]. Let $\alpha, \alpha(A), M, \beta$ and $L$ be the constant defined by (17), (26), (28), (30) and (68) respectively. Let $n_{0}$ and $n_{1}$ be positive integers satisfying conditions (43) and (40). Let $\widetilde{\lambda}_{n}$ be the $n_{1}$-first approximate roots of the equation (18), each one in the interval $(n \pi,(n+1) \pi), n \leq n_{1}$, and let $\tilde{\lambda}_{0}$ be the approximation of the additional solution $\lambda_{0} \in(0, \pi)$ to be consider if condition (19) holds. Let $\gamma>0$ be satisfying (53) and let $\Lambda, \mathcal{A}, \mathcal{B}$ and $L^{\star}$ be the positive constants defined by (63), (64) and (68) respectively. Suppose that the approximations $\widetilde{\lambda_{n}}$ satisfy (71), where $S$ is the constant defined by (70). Suppose that the approximations App $\left(\mathrm{e}^{-{\widetilde{\lambda_{n}}}^{2} A t}\right)$ of matrices $\mathrm{e}^{-\widetilde{\lambda}_{n}^{2} A t}$, for $n \leq n_{1}$ satisfy that the approximation error is less than $\mathcal{K}$, where $\mathcal{K}$ is a positive constant which satisfies (77). Consider the functions $X_{\widetilde{\lambda_{n}}}(x)$, $n \leq n_{1}$ defined by (45) and vectors $C\left(\widetilde{\lambda_{n}}\right), n \leq n_{1}$, defined by (46), joint the vector $C(0)$ defined by (24) if $\alpha \neq 0$. Then, the vector valued function $\mathcal{U}\left(x, t, n_{1}\right)$ defined by (75) satisfies

$$
\left\|u(x, t)-\mathcal{U}\left(x, t, n_{1}\right)\right\|^{2} \leq \varepsilon, \quad(x, t) \in D\left[t_{0}, t_{1}\right]
$$

Theorem 2. We consider problem (1)-(4) satisfying hypotheses (5), (6) and (7). Let $\varepsilon>0$, and we consider the subdomain $D\left[t_{0}, t_{1}\right]=[0,1] \times\left[t_{0}, t_{1}\right]$. Suppose that the hypothesis $(b)$ is verified, this ensures that there is an exact solution $u(x, t)$ of problem (1)-(4), see Ref. [16]. Let $\alpha, \alpha(A), M$ and L be the constant defined by (20), (26), (28) and (68) respectively. Let $n_{0}$ and $n_{1}$ be positive integers satisfying conditions (43) and (40). Take $\beta=1$ and $b_{1}=0$. Let $\tilde{\lambda}_{n}$ be the $n_{1}$-first approximate roots of the equation (21), each one in the interval $(n \pi,(n+1) \pi), n \leq n_{1}$, and let $\widetilde{\lambda}_{0}$ be the approximation of the additional solution $\lambda_{0} \in(0, \pi)$ to be consider if condition (22) holds. Let $\gamma>0$ be satisfying (53) and let $\Lambda, \mathcal{A}, \mathcal{B}$ and $L^{\star}$ be the positive constants defined by (63), (64) and (68) respectively. Suppose that the approximations $\tilde{\lambda}_{n}$ satisfy (71), where $S$ is the constant defined by (70). Suppose that the approximations $\operatorname{App}\left(\mathrm{e}^{-{\widetilde{\lambda_{n}}}^{2} A t}\right)$ of matrices $\mathrm{e}^{-{\widetilde{\lambda_{n}}}^{2} A t}$, for $n \leq n_{1}$ satisfy that the approximation error is less than $\mathcal{K}$, where $\mathcal{K}$ is a positive constant which satisfies (77). Consider the functions $X_{\widetilde{\lambda}_{n}}(x), n \leq n_{1}$ defined by (45) and vectors $C\left(\widetilde{\lambda}_{n}\right), n \leq n_{1}$, defined by (46), joint the vector $C(0)$ defined by (24) if $\alpha \neq 0$. Then, the vector valued function $\mathcal{U}\left(x, t, n_{1}\right)$ defined by (75) satisfies

$$
\left\|u(x, t)-\mathcal{U}\left(x, t, n_{1}\right)\right\|^{2} \leq \varepsilon, \quad(x, t) \in D\left[t_{0}, t_{1}\right]
$$

\section{Algorithm 1, Algorithm 2 and Example}

We can give the following algorithms, according to the hypothesis (a) or (b) is satisfied, to construct the approximation $\mathcal{U}\left(x, t, n_{1}\right)$. 
Algorithm 1. Construction of the analytic-numerical solution of problem (1)-(4) under hypotheses (a) in the subdomain $D\left[t_{0}, t_{1}\right]=[0,1] \times\left[t_{0}, t_{1}\right], \quad t_{0}>0$, with a priori error bound $\varepsilon>0$.

1: Compute the constant $\rho_{0}$ satisfying (7).

2: Determine $b_{1}$ and $b_{2}$ satisfying (10). Compute constant $\alpha$ defined by (17).

3: Compute constants $\|A\|, \alpha(A), \quad M, \beta, L$ defined by (26), (28), (30) and (68) respectively.

4: Determine the first positive integer $n_{0}$ which satisfies (43).

5: Determine the first positive integer $n_{1}$ which satisfies (40).

6: Determine approaches $\tilde{\lambda}_{n}$ of the $n_{1}$-first roots of Equation (18) each one in the interval $(k \pi,(k+1) \pi), \quad k \leq n_{1}$, joint the approximation of the additional solution $\lambda_{0} \in(0, \pi)$ if condition (19) holds.

7: Compute $I(\rho)$ for $\rho=\widetilde{\lambda}_{n}, n \leq n_{1}$ and determine $\gamma>0$ satisfying (53).

8: Compute $\Lambda, \mathcal{A}, \mathcal{B}$ and $L^{\star}$ defined by (63), (64) and (68) respectively.

9: Compute $S$ defined by (70)

10: Check that approximations $\quad \tilde{\lambda}_{n}$ satisfy (71). Otherwise return to step 6 and calculate approximations $\quad \widetilde{\lambda}_{n}$ more precisely.

11: Compute $\mathcal{K}$ satisfying (77).

12: Compute approximations $\operatorname{App}\left(\mathrm{e}^{-{\widetilde{\lambda_{n}}}^{2} A t}\right)$ of matrices $\mathrm{e}^{-\widetilde{\lambda}_{n}^{2} A t}$, for $n \leq n_{1}$ so that the error in each one approach is less than $\mathcal{K}$.

13: Compute functions $X_{\widetilde{\pi}_{n}}(x), n \leq n_{1}$, defined by (45).

14: Compute vectors $C\left(\widetilde{\lambda}_{n}\right), n \leq n_{1}$, defined by (46). If $\alpha \neq 0$, compute $C(0)$ defined by (24).

15: Compute the approximation $\mathcal{U}\left(x, t, n_{1}\right)$ defined by (75).

Algorithm 2. Construction of the analytic-numerical solution of problem (1)-(4) under hypotheses (b) in the subdomain $D\left[t_{0}, t_{1}\right]=[0,1] \times\left[t_{0}, t_{1}\right], t_{0}>0$, with a priori error bound $\varepsilon>0$.

1: Compute the constant $\rho_{0}$ satisfying (7).

2: Determine $a_{2}$ satisfying (13). Compute constant $\alpha$ defined by (20). Take $b_{1}=0$ and $\beta=1$.

3: Compute constants $\|A\|, \alpha(A), \quad M, L$ defined by (26), (28) and (68) respectively.

4: Determine the first positive integer $n_{0}$ which satisfies (43).

5: Determine the first positive integer $n_{1}$ which satisfies (40).

6: Determine approaches $\widetilde{\lambda}_{n}$ of the $n_{1}$-first roots of Equation (21) each one in the interval $(k \pi,(k+1) \pi), \quad k \leq n_{1}$, joint the approximation of the additional solution $\lambda_{0} \in(0, \pi)$ if condition (22) holds.

\section{Continue with the step 7 of Algorithm 1}

Example 1. We will construct an approximate solution in the subdomain $D[0,1]=[0,1] \times[0.1,1]$, with a priori error bound $\varepsilon=10^{-2}$, of the homogeneous parabolic problem with homogeneous conditions (1)-(4), where the matrix $A \in \mathbb{C}^{4 \times 4}$ is chosen

$$
A=\left(\begin{array}{cccc}
2 & 0 & 0 & -1 \\
1 & 2 & 1 & -2 \\
-1 & 0 & 2 & 1 \\
0 & 0 & 0 & 1
\end{array}\right)
$$

and the $4 \times 4$ matrices $A_{i}, B_{i}, i \in\{1,2\}$, are 


$$
\begin{array}{ll}
A_{1}=\left(\begin{array}{llll}
0 & 0 & 0 & 0 \\
0 & 0 & 0 & 0 \\
0 & 0 & 1 & 0 \\
0 & 0 & 0 & 1
\end{array}\right), & A_{2}=\left(\begin{array}{llll}
0 & 1 & 0 & 0 \\
1 & 0 & 0 & 0 \\
0 & 0 & 0 & 1 \\
0 & 0 & 0 & 0
\end{array}\right), \\
B_{1}=\left(\begin{array}{llll}
1 & 0 & 0 & 0 \\
0 & 1 & 0 & 0 \\
0 & 0 & 0 & 0 \\
0 & 0 & 0 & 0
\end{array}\right), & B_{2}=\left(\begin{array}{llll}
1 & 0 & 0 & 0 \\
1 & 0 & 0 & 0 \\
0 & 0 & 1 & 0 \\
0 & 0 & 0 & 1
\end{array}\right)
\end{array}
$$

Also, the vectorial valued function $f(x)$ will be defined as

$$
f(x)=\left(\begin{array}{c}
0 \\
x^{2}-1 \\
0 \\
0
\end{array}\right)
$$

This is precisely the example 1 of Ref. [15] whose exact solution is given by:

$$
u(x, t)=\left(\sum_{n \geq 0}-\frac{32(-1)^{n} \mathrm{e}^{-\frac{\pi}{2}(2 n+1)^{2} t} \cos \left(\frac{\pi}{2}(2 n+1) x\right)}{\pi^{3}(2 n+1)^{3}}\right)\left(\begin{array}{l}
0 \\
1 \\
0 \\
0
\end{array}\right)
$$

We will follow algorithm 1 step by step:

1. Hypothesis (a) holds with $m=4$. Note that although $A_{1}$ is singular, taking $\rho_{0}=1 \in \mathbb{R}$, the matrix pencil

$$
A_{1}+\rho_{0} B_{1}=I_{4 \times 4}
$$

is regular. Therefore, we take $\rho_{0}=1$.

2. Performing calculations similar to those made in Ref. [15], one gets that $b_{1}=1, b_{2}=0$ and $\alpha=0$.

3. It is easy to calculate $\|A\|=3.67571, \sigma(A)=\{1,2\}$, thus $\alpha(A)=2$. Similarly $M=8 / 15, \beta=1$ and $L=101.589$.

4. Note that

$$
\frac{1}{M \beta L^{2}}=0.00018168 \text {. }
$$

Then, by (43):

$$
\begin{aligned}
& n=3 \Rightarrow(3 \pi)^{16} \mathrm{e}^{-0.4\left(3^{2}\right) \pi^{2}}=1.43749>\frac{1}{M \beta L^{2}}, \\
& n=4 \Rightarrow(4 \pi)^{16} \mathrm{e}^{-0.4\left(4^{2}\right) \pi^{2}}=1.428708 \times 10^{-10}<\frac{1}{M \beta L^{2}},
\end{aligned}
$$

then we take $n_{0}=4$.

5. We have

$$
\begin{aligned}
& n=4 \Rightarrow\left(\sum_{k=1}^{4} \frac{1}{k^{4}}-\frac{\pi^{4}}{90}+\frac{10^{-2}}{3}\right)=-0.000237971, \\
& n=5 \Rightarrow\left(\sum_{k=1}^{5} \frac{1}{k^{4}}-\frac{\pi^{4}}{90}+\frac{10^{-2}}{3}\right)=0.00136203,
\end{aligned}
$$

then we can take $n_{1}=5>n_{0}=4$.

6 . We need to determinate the $n_{1}$-first roots of equation 


$$
\lambda \cot (\lambda)=0
$$

We can solve exactly this equation, $\lambda_{n}=\frac{\pi}{2}+n \pi, n=1, \cdots, 5$, with an additional solution $\left.\lambda_{0} \in\right] 0, \pi[$, because

$$
\frac{\left(1-b_{2}+\rho_{0} b_{1} b_{2}\right)\left(1-\rho_{0} b_{1}\right)}{b_{1}}=0<1
$$

and then $\lambda_{0}=\frac{\pi}{2}$.

In summary, $\lambda_{0}=\frac{\pi}{2}, \lambda_{1}=\frac{3 \pi}{2}, \lambda_{2}=\frac{5 \pi}{2}, \lambda_{3}=\frac{7 \pi}{2}, \lambda_{4}=\frac{9 \pi}{2}, \lambda_{5}=\frac{11 \pi}{2}$. We take the approximate values (50 exact decimal)

$$
\begin{aligned}
& \widetilde{\lambda_{0}}=1.5707963267948966192313216916397514420985846996876, \\
& \widetilde{\lambda}_{1}=4.7123889803846898576939650749192543262957540990627, \\
& \widetilde{\lambda_{2}}=7.8539816339744830961566084581987572104929234984378, \\
& \widetilde{\lambda_{3}}=10.995574287564276334619251841478260094690092897813, \\
& \widetilde{\lambda_{4}}=14.137166941154069573081895224757762978887262297188, \\
& \widetilde{\lambda_{5}}=17.278759594743862811544538608037265863084431696563 .
\end{aligned}
$$

7. We calculate $I(\rho)$ for $\rho=\widetilde{\lambda_{n}}$ :

$$
\begin{aligned}
& I\left(\tilde{\lambda}_{0}\right)=1.2337005501361698273543113749845188919142124259051, \\
& I\left(\widetilde{\lambda}_{1}\right)=11.103304951225528446188802374860670027227911833146, \\
& I\left(\tilde{\lambda}_{2}\right)=30.842513753404245683857784374612972297855310647627, \\
& I\left(\widetilde{\lambda}_{3}\right)=60.451326956672321540361257374241425703796408869350, \\
& I\left(\widetilde{\lambda}_{4}\right)=99.929744561029756015699221373746030245051206498313, \\
& I\left(\widetilde{\lambda}_{5}\right)=149.27776656647654910987167637312678592161970353452 .
\end{aligned}
$$

the smallest of them is $I\left(\widetilde{\lambda_{0}}\right)$, as $1 / \widetilde{\lambda_{0}} \approx 0.810569$, we take $\gamma=0.82$.

8. We have that $\Lambda=17.3>\widetilde{\lambda_{5}}, \mathcal{A}=18.3, \mathcal{B}=34.6$ and $L^{\star}=1.77759 \times 10^{9}$.

9. We have that $S=1.56631 \times 10^{43}$.

10. To be applicable the algorithm 1 , the approximations $\widetilde{\lambda}_{n}$ may satisfy:

$$
\left|\lambda_{n}-\widetilde{\lambda}_{n}\right|<\min \left\{1, \frac{\sqrt{\varepsilon}}{\sqrt{3} n_{1} S}\right\}=7.37211 \times 10^{-46}
$$

As the roots were calculated with 50 decimal accurate, we accept these approximations of the roots.

11 . We have to take $\mathcal{K}$ satisfying (77). In our case

$$
\mathcal{K}<\frac{\sqrt{\varepsilon}}{\sqrt{3} n_{1} t_{1} \gamma \mathcal{A}^{2} \sqrt{M}}=0.0000472137 .
$$

12. We have to compute approximations $\operatorname{App}\left(\mathrm{e}^{-\lambda_{n}^{2} A t}\right)$ of matrices $\mathrm{e}^{-\lambda_{n}^{2} A t}$, for $n=0,1,2,3,4,5$ with a maximum error $\mathcal{K}$. In this case, using minimal theorem ([28], p. 571), we can determine the exact value of $\mathrm{e}^{A s}$ given by: 


$$
\mathrm{e}^{A s}=\left(\begin{array}{cccc}
\mathrm{e}^{-2 s} & 0 & 0 & \mathrm{e}^{-2 s}\left(-1+\mathrm{e}^{s}\right) \\
-\frac{1}{2} \mathrm{e}^{-2 s} s(2+s) & \mathrm{e}^{-2 s} & -\mathrm{e}^{-2 s} s & \frac{1}{2} \mathrm{e}^{-2 s}\left(-2+2 \mathrm{e}^{s}+2 s+s^{2}\right) \\
\mathrm{e}^{-2 s} s & 0 & \mathrm{e}^{-2 s} & -\mathrm{e}^{-2 s} s \\
0 & 0 & 0 & \mathrm{e}^{-s}
\end{array}\right)
$$

then, we can obtain $\operatorname{App}\left(\mathrm{e}^{-{\widetilde{\lambda_{n}}}^{2} A t}\right)$ for $n=0,1,2,3,4,5$ replacing in (84).

13. Functions $X_{\widetilde{\lambda_{n}}}(x), n=0,1, \cdots, 4$, defined by (45) are given by:

$$
\begin{aligned}
& X_{\widetilde{\lambda_{0}}}(x)=-1.5707963267948966192 \cos (1.5707963267948966192 x), \\
& X_{\widetilde{\lambda_{1}}}(x)=-4.7123889803846898577 \cos (4.7123889803846898577 x), \\
& X_{\widetilde{\lambda_{2}}}(x)=-7.8539816339744830962 \cos (7.8539816339744830962 x), \\
& X_{\widetilde{\lambda_{3}}}(x)=-10.995574287564276335 \cos (10.995574287564276335 x), \\
& X_{\widetilde{\lambda_{4}}}(x)=-14.137166941154069573 \cos (14.137166941154069573 x), \\
& X_{\widetilde{\lambda_{5}}}(x)=-17.278759594743862812 \cos (17.278759594743862812 x) .
\end{aligned}
$$

14. Vectors $C\left(\widetilde{\lambda_{n}}\right), n=1, \cdots, 5$, defined by (46) are given by:

$$
\begin{aligned}
& C\left(\widetilde{\lambda}_{0}\right)=\left(\begin{array}{c}
0 \\
0.65702286429979745210577812909559642508 \\
0 \\
0
\end{array}\right), \\
& C\left(\widetilde{\lambda}_{1}\right)=\left(\begin{array}{c}
-0.0081113933864172524951330633221678570997 \\
0 \\
0 \\
0
\end{array}\right), \\
& C\left(\widetilde{\lambda}_{2}\right)=\left(\begin{array}{c}
0.00105123658287967592336924500655295428012 \\
0 \\
0 \\
0
\end{array}\right), \\
& C\left(\widetilde{\lambda}_{3}\right)=\left(\begin{array}{c}
-0.0002736455078299864440257301662205732716 \\
0 \\
0
\end{array}\right), \\
& C\left(\widetilde{\lambda}_{5}\right)=\left(\begin{array}{c}
0 \\
0.0001001406590915710184584328805205908284 \\
0 \\
0 \\
0.00004487554567992606052221693389082688512 \\
0 \\
0
\end{array}\right),
\end{aligned}
$$


We don't compute $C(0)$ defined by (25) because $\alpha=0$.

15. Compute $\mathcal{U}\left(x, t, n_{1}\right)$ defined by (75), obtaining:

$$
\mathcal{U}(x, t, 5)=\left(\begin{array}{c}
0 \\
\mathcal{W}(x, t) \\
0 \\
0
\end{array}\right)
$$

where

$$
\begin{aligned}
\mathcal{W}(x, t)= & -1.03204910 \mathrm{e}^{-4.93480220 t} \cos (1.57079633 x)+0.0382240408 \mathrm{e}^{-44.4132198 t} \cos (4.71238898 x) \\
& -0.00825639281 \mathrm{e}^{-123.370055 t} \cos (7.85398163 x)+0.00300888951 \mathrm{e}^{-241.805308 t} \cos (10.9955743 x) \\
& -0.00141570522 \mathrm{e}^{-399.718978 t} \cos (14.1371669 x)+0.000775393765 \mathrm{e}^{-597.111066 t} \cos (17.2787596 x) .
\end{aligned}
$$

and our approximation satisfies

$$
\|u(x, t)-\mathcal{U}(x, t, 5)\|^{2}<10^{-2}, \quad(x, y) \in D[0.1,1]
$$

As an example, consider the point $(x, t)=(0.27,0.9) \in D[0.1,1]$. We have the approximation

$$
\mathcal{U}(0.27,0.9,5)=\left(\begin{array}{c}
0 \\
-0.0110808 \\
0 \\
0
\end{array}\right)
$$

It is easy to check that, from (82), one gets

$$
\|u(0.27,0.9)-\mathcal{U}(0.27,0.9,5)\|<10^{-18}
$$

\section{Conclusion}

In this paper, a method to construct an analytic-numerical solution for homogeneous parabolic coupled systems with homogeneous boundary conditions of the type (1)-(4) has been presented. An algorithm with an illustrative example is given.

\section{References}

[1] Alexander, M.H. and Manolopoulos, D.E. (1987) A Stable Linear Reference Potencial Algorithm for Solution of the Quantum Close-Coupled Equations in Molecular Scattering Theory. The Journal of Chemical Physics, 86, 2044-2050. http://dx.doi.org/10.1063/1.452154

[2] Melezhik, V.S., Puzynin, I.V., Puzynina, T.P. and Somov, L.N. (1984) Numerical Solution of a System of Integro-Differential Equations Arising from the Quantum-Mechanical Three-Body Problem with Coulomb Interaction. Journal of Computational Physics, 54, 221-236. http://dx.doi.org/10.1016/0021-9991(84)90115-3

[3] Reid, W.T. (1971) Ordinary Differential Equations. Wiley, New York.

[4] Levine, R.D., Shapiro, M. and Johnson, B. (1970) Transition Probabilities in Molecular Collisions: Computational Studies of Rotational Excitation. The Journal of Chemical Physics, 53, 1755-1766. http://dx.doi.org/10.1063/1.1673214

[5] Schmalz, T.G., Lill, J.V. and Light, J.C. (1983) Imbedded Matrix Green's Functions in Atomic and Molecular Scattering theory. The Journal of Chemical Physics, 78, 4456-4463. http://dx.doi.org/10.1063/1.445338

[6] Mrugala, F. and Secrest, D. (1983) The Generalized Log-Derivate Method for Inelastic and Reactive Collisions. The Journal of Chemical Physics, 78, 5954-5961. http://dx.doi.org/10.1063/1.444610

[7] Crank, J. (1995) The Mathematics of Diffusion. 2nd Edition, Oxford University Press, Oxford.

[8] Mikhailov, M.D. and Osizik, M.N. (1984) Unifield Analysis and Solutions of Heat and Mass Diffusion. Wiley, New York. 
[9] Stakgold, I. (1979) Green’s Functions and Boundary Value Problems. Wiley, New York.

[10] Hueckel, T., Borsetto, M. and Peano, A. (1987) Modelling of Coupled Thermo-Elastoplastic Hydraulic Response of clays Subjected to Nuclear Waste Heat. Wiley, New York.

[11] Atkinson, F.V. (1964) Discrete and Continuous Boundary Value Problems. Academic Press, New York.

[12] Atkinson, F.V., Krall, A.M., Leaf, G.K. and Zettel, A. (1987) On the Numerical Computation of Eigenvalues of Sturm? Liouville Problems with Matrix Coefficients. Technical Report, Argonne National Laboratory.

[13] Marletta, M. (1991) Theory and Implementation of Algorithms for Sturm-Liouville Systems. Ph.D. Thesis, Royal Military College of Science, Cranfield.

[14] Greenberg, L. (1991) A Prüfer Method for Calculating Eigenvalues of Self-Adjoint Systems of Ordinary Differential Equations, Parts 1 and 2. Technical Report TR91-24, University of Maryland.

[15] Soler, V., Defez, E., Ferrer, M.V. and Camacho, J. (2013) On Exact Series Solution of Strongly Coupled Mixed Parabolic Problems. Abstract and Applied Analysis, 2013, Article ID: 524514.

[16] Soler, V., Defez, E. and Verdoy, J.A. (2014) On Exact Series Solution for Strongly Coupled Mixed Parabolic Boundary Value Problems. Abstract and Applied Analysis, 2014, Article ID: 759427.

[17] Campbell, S.L. and Meyer Jr., C.D. (1979) Generalized Inverses of Linear Transformations. Pitman, London.

[18] Navarro, E., Jódar, L. and Ferrer, M.V. (2002) Constructing Eigenfunctions of Strongly Coupled Parabolic Boundary Value Systems. Applied Mathematical Letters, 15, 429-434. http://dx.doi.org/10.1016/S0893-9659(01)00154-9

[19] Pryce, J.D. (1993) Numerical Solution of Sturm-Liouville Problems. Clarendon, Oxford.

[20] Pryce, J.D. and Marletta, M. (1992) Automatic Solution of Sturm-Liouville Problems Using Pruess Method. Journal of Computational and Applied Mathematics, 39, 57-78. http://dx.doi.org/10.1016/0377-0427(92)90222-J

[21] Moler, C.B. and Van Loan, C.F. (1978) Nineteen Dubious Ways to Compute the Exponential of a Matrix. SIAM Review, 20, 801-836. http://dx.doi.org/10.1137/1020098

[22] Moler, C.B. and Van Loan, C.F. (2003) Nineteen Dubious Ways to Compute the Exponential of a Matrix, Twenty-Five Years Later. SIAM Review, 45, 3-49.

[23] Golub, G.H. and Van Loan, C.F. (1989) Matrix Computation. The Johns Hopkins University Press, Baltimore.

[24] Coddington, E.A. and Levinson, N. (1967) Theory of Ordinary Differential Equations. McGraw-Hill, New York.

[25] Sastre, J., Ibáñez, J., Ruiz, P. and Defez, E. (2014) Accurate and Efficient Matrix Exponential Computation. International Journal of Computer Mathematics, 91, 97-112. http://dx.doi.org/10.1080/00207160.2013.791392

[26] Sastre, J., Ibáñez, J., Defez, E. and Ruiz, P. (2011) Accurate Matrix Exponential Computation to Solve Coupled Differential Models in Engineering. Mathematical and Computer Modelling, 54, 1835-1840. http://dx.doi.org/10.1016/j.mcm.2010.12.049

[27] Sastre, J., Ibáñez, J., Defez, E. and Ruiz, P. (2011) Efficient Orthogonal Matrix Polynomial Based Method for Computing Matrix Exponential. Applied Mathematics and Computation, 217, 6451-6463.

[28] Dunford, N. and Schwartz, J. (1977) Linear Operators, Part I. Interscience, New York. 
Scientific Research Publishing (SCIRP) is one of the largest Open Access journal publishers. It is currently publishing more than 200 open access, online, peer-reviewed journals covering a wide range of academic disciplines. SCIRP serves the worldwide academic communities and contributes to the progress and application of science with its publication.

Other selected journals from SCIRP are listed as below. Submit your manuscript to us via either submit@scirp.org or Online Submission Portal.
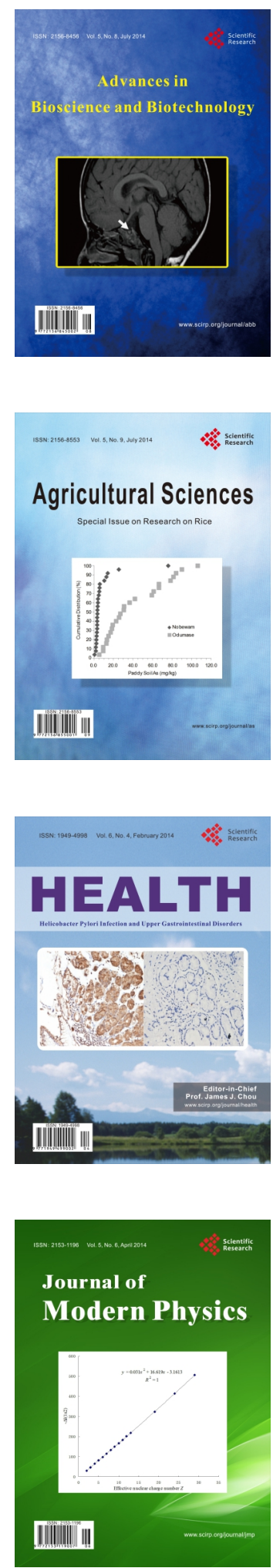
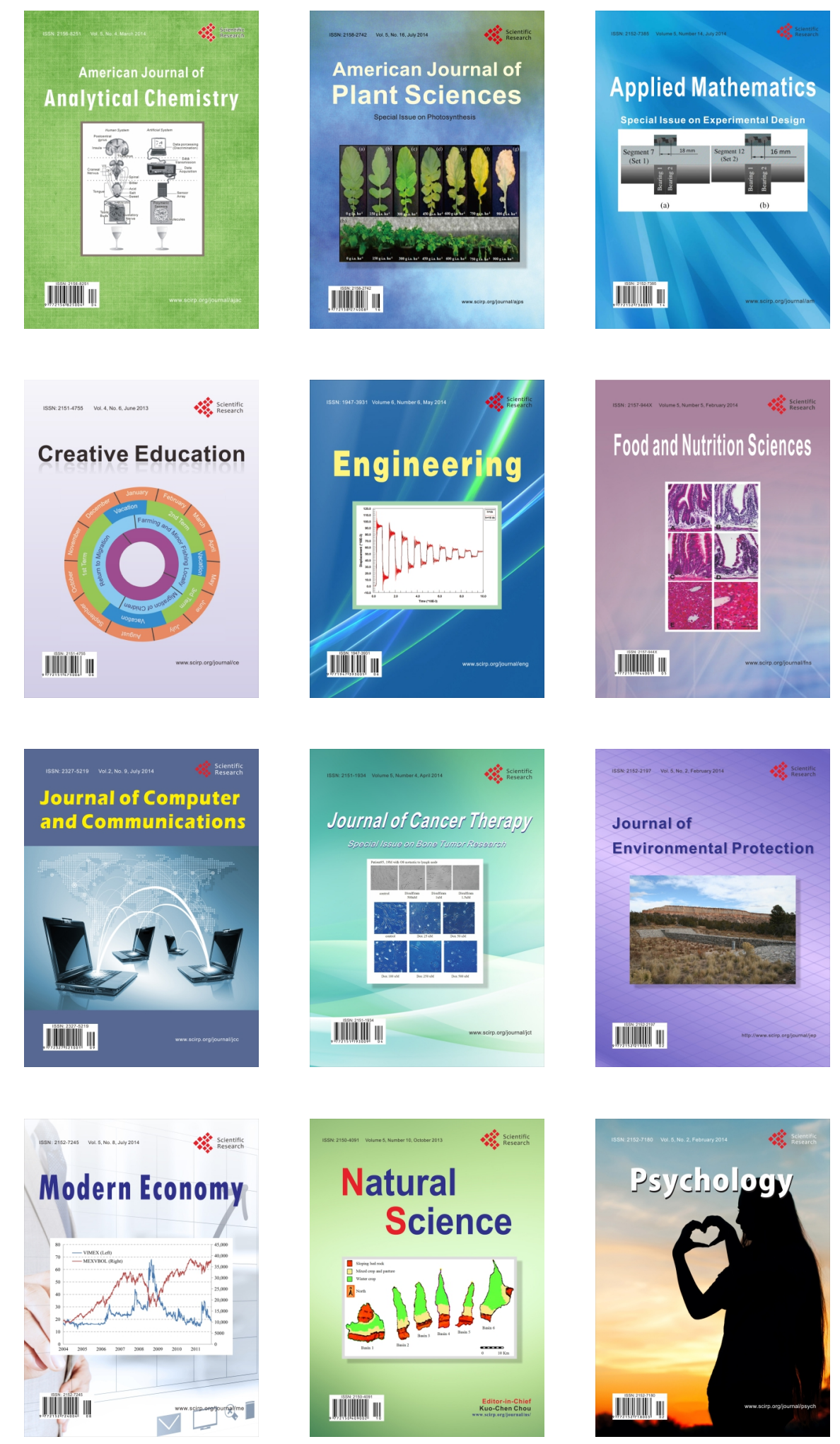